\title{
Review of: "One-step construction of circularized nanodiscs using SpyCatcher-SpyTag"
}

\section{Chang Sun}

Potential competing interests: The author(s) declared that no potential competing interests exist.

This article shows an interesting application of the isopeptide bond between the SpyCatcher and the Spy tag--turning linear membrane scaffold protein (MSP) into cyclic. To this end, the authors have carried out a systematic study covering a range of MSPs from $10 \mathrm{~nm}$ to $100 \mathrm{~nm}$ and documented the whole process from protein expression, purification, to nanodisc formation.

While the results are mostly successful, particularly for the large MSPs which are known to be hard to work with, the reconstituted cyclic nanodiscs failed to demonstrate improved heterogeneity compared to their linear counterparts. Notably in Figure 2, the SEC traces of the circularized MSP1D1, which I have worked with the linear counterpart, showed substantial heterogeneity and appeared to be prone to aggregation. One possible explanation is the inclusion of the Spycatcher that is not typically amphipathic causes unfavorable interactions with phospholipids. In short, the new application enabled production of large cyclic MSPs, at the cost of reduced nanodisc homogeneity.

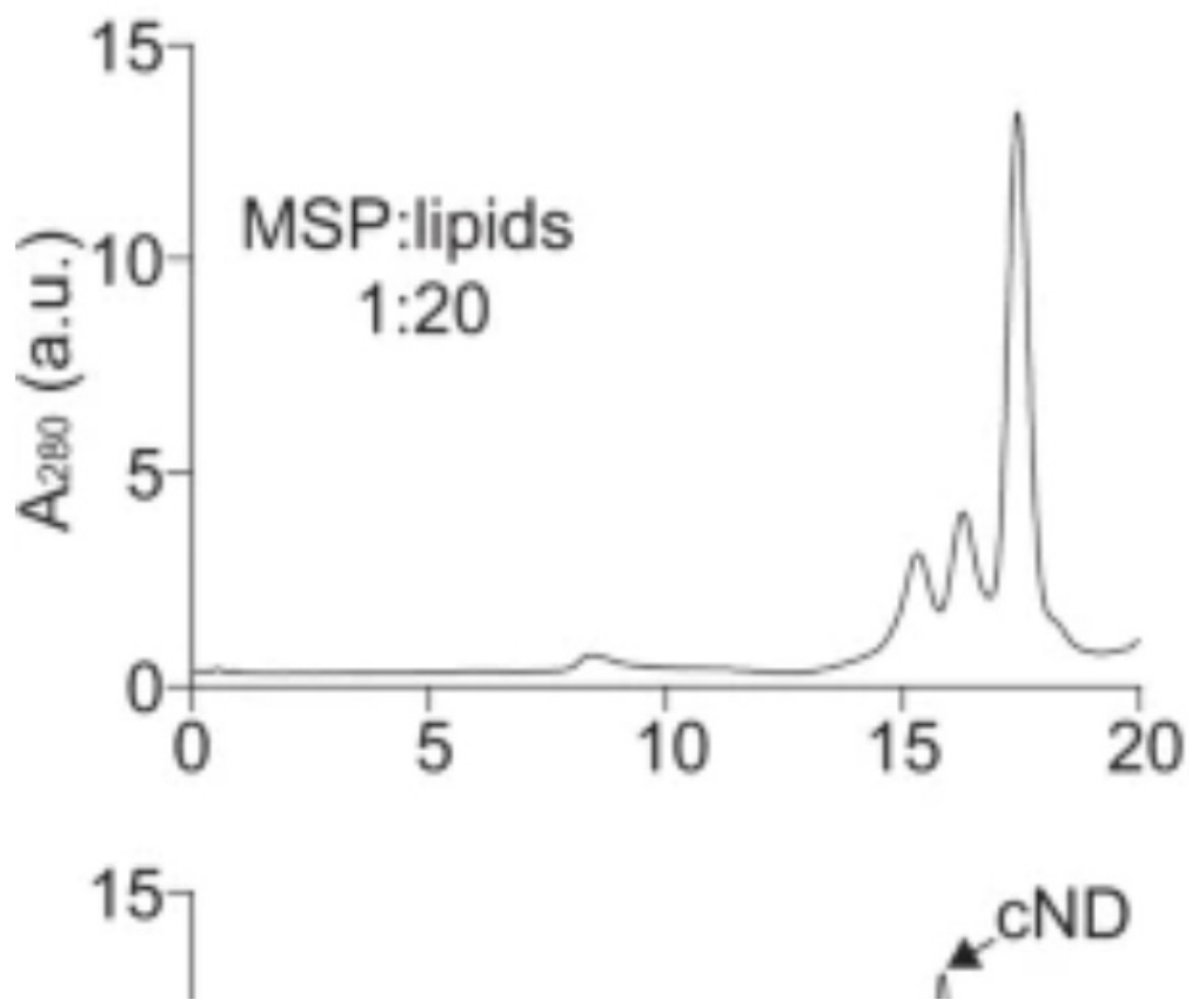



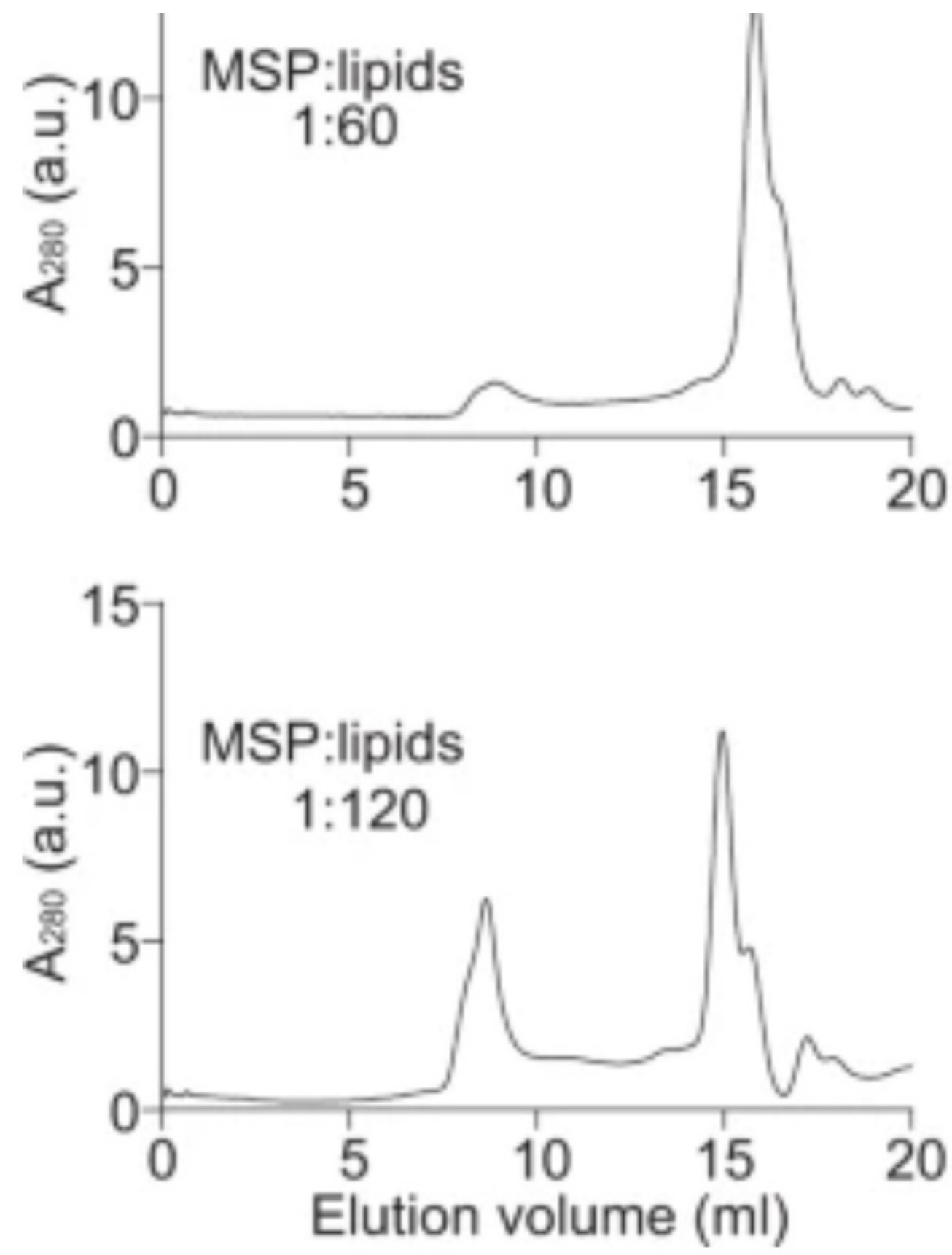

Another critique of this paper is the omission of the work from N. T. Johansen et al. (FEBS 2019) where cyclic MSPs were produced using an optimized Sortase protocol from a re-designed MSP, which produced homogenous cyclic MSP nanodiscs with a very high protein yield. 